\title{
Low-Dose Aspirin
}

National Cancer Institute

\section{Source}

National Cancer Institute. Low-Dose Aspirin. NCI Thesaurus. Code C94758.

Aspirin containing a lower dose than a standard adult tablet, usually between 81 and $100 \mathrm{mg}$. 